\title{
Effects of Cornell-type Potential on Klein-Gordon Oscillator Under a Linear Central Potential Induced by Lorentz Symmetry Violation
}

\author{
Faizuddin Ahmed ${ }^{1}$ \\ National Academy Gauripur, Assam, 783331, India
}

\begin{abstract}
In this work, we study a Klein-Gordon oscillator subject to Cornelltype potential in the background of the Lorentz symmetry violation determined by a tensor out of the Standard Model Extension. We introduce a Cornell-type potential $S(r)=\left(\eta_{L} r+\frac{\eta_{c}}{r}\right)$ by modifying the mass term via transformation $M \rightarrow M+S(r)$ and then coupled oscillator with scalar particle by replacing the momentum operator $\vec{p} \rightarrow(\vec{p}+i M \omega \vec{r})$ in the relativistic wave equation. We see that the analytical solution to the Klein-Gordon oscillator equation can be achieved, and a quantum effect characterized by the dependence of the angular frequency of the oscillator on the quantum numbers of the relativistic system is observed.
\end{abstract}

Keywords: Lorentz symmetry violation, Relativistic wave-equations: bound states solutions, scalar potential, electric \& magnetic field, biconfluent Heun's function .

PACS Number(s): 03.65.Pm, 11.30.Cp, 11.30.Qc

\section{Introduction}

The Klein-Gordon oscillator [1, 2] inspired by the Dirac oscillator [3] has been studied in the quantum mechanics $[4,5,6,7,8,9,10,11,12,13]$. Several authors have been used different kind of potentials to study the Klein-Gordon

\footnotetext{
${ }^{1}$ faizuddinahmed15@gmail.com ; faiz4U.enter@rediffmail.com
} 
oscillator in the space-time background (see, Ref. [12, 13] and related references their in). In this work, we study the Klein-Gordon oscillator under the effects of violation of the Lorentz symmetry defined by a tensor $\left(K_{F}\right)_{\mu \nu \alpha \beta}$ out of the Standard Model Extension in the presence of a scalar potential. The gauge sector of the Standard Model Extension [14, 15, 16, 17, 18, 19] consists two violating terms that modifies the transport properties of space-time since these terms break the Lorentz symmetry. These two terms are called the CPT-odd sector [17, 18] and the CPT-even sector [19]. In relativistic quantum system, several authors investigated the relativistic wave equations under Lorentz symmetry breaking effects with or without potential of various kind $[20,21,22,23,24,25,26,28,29]$.

The relativistic quantum dynamics of spin-0 particle under the effects of the Lorentz symmetry violation [20, 21, 22, 23, 24, 25, 26, 27, 28, 29, 30]

$$
p^{\mu} p_{\mu} \Psi+\frac{\alpha}{4}\left(K_{F}\right)_{\mu \nu \alpha \beta} F^{\mu \nu}(x) F^{\alpha \beta}(x) \Psi=M^{2} \Psi,
$$

where $\alpha$ is a constant, $F_{\mu \nu}(x)=\partial_{\mu} A_{\nu}-\partial_{\nu} A_{\mu}$ is the electromagnetic tensor, $\left(K_{F}\right)_{\mu \nu \alpha \beta}$ corresponds to a tensor that governs the Lorentz symmetry violation out of the Standard Model Extension.

One can introduce a non-electromagnetic (scalar) potential in the wave equation by modifying the mass term $M \rightarrow M+S(r)[31,32]$, where $S(r)$ is the static scalar potential. Therefore, the modified Klein-Gordon equation (1) becomes

$$
p^{\mu} p_{\mu} \Psi+\frac{\alpha}{4}\left(K_{F}\right)_{\mu \nu \alpha \beta} F^{\mu \nu}(x) F^{\alpha \beta}(x) \Psi=(M+S(r))^{2} \Psi .
$$

The Cornell-type potential in cylindrical system [6, 33, 32] has growing interest in theoretical physics. This type of potential includes two conceptsconfinement and asymptotic freedom. The Coulomb-like part is responsible for small distances interactions whereas linear potential term is a long range that leads to the confinement phenomena of quarks. This type of potential has been studied in the relativistic quantum system [7, 9, 34, 35, 36, 37], 
bound state of hadrons [38, 39], and the ground state of three quarks [40] in particle physics. This type of potential is given by [30]

$$
S(r)=\left(\eta_{L} r+\frac{\eta_{c}}{r}\right)
$$

where $\eta_{L}>0, \eta_{c}>0$ are arbitrary constants.

Further, if one includes oscillator with Klein-Gordon field, following change in the momentum operator is considered [10, 13, 4, 5, 6, 7, 8, 9, 11, 12, 27, 41]:

$$
\vec{p} \rightarrow(\vec{p}+i M \omega \vec{r}) \quad \text { or } \quad \frac{\partial}{\partial r} \rightarrow\left(\frac{\partial}{\partial r}-M \omega r\right)
$$

where $\omega$ is the oscillator frequency and $\vec{r}=r \hat{r}$ with $r$ being the distance from the particle to the axis. So we can write $\vec{p}^{2} \rightarrow(\vec{p}+i M \omega \vec{r})(\vec{p}-i M \omega \vec{r})$.

Therefore, the modified Klein-Gordon oscillator from (1) becomes

$$
\begin{aligned}
& {\left[\left(p^{\mu}+i M \omega X^{\mu}\right)\left(p_{\mu}-i M \omega X_{\mu}\right)+\frac{\alpha}{4}\left(K_{F}\right)_{\mu \nu \alpha \beta} F^{\mu \nu}(x) F^{\alpha \beta}(x)\right] \Psi} \\
& =(M+S(r))^{2} \Psi
\end{aligned}
$$

where $X_{\mu}=(0, r, 0,0)$. Note that the Klein-Gordon oscillator under a linear central potential induced by the Lorentz symmetry violation in the presence of a Cornell-type potential hasn't yet been investigated which is our aim in this paper.

The structure of this paper is as follows: in section 2, we introduce Lorentz symmetry violation defined by a tensor $\left(K_{F}\right)_{\mu \nu \alpha \beta}$ that governs the Lorentz symmetry breaking effects out of the Standard Model Extension. Then we inserted a Cornell-type scalar potential by modifying the mass term in the wave equation and analyze the effects on eigenvalues and wave function; in section 3, we present our conclusions. 


\section{Relativistic oscillator particle under LSV effects subject to Cornell-type potential}

The modified Klein-Gordon oscillator equation (5) in the background of the Minkowski space-time becomes

$$
\begin{aligned}
& {\left[-\frac{\partial^{2}}{\partial t^{2}}+\frac{1}{r}\left(\frac{\partial}{\partial r}+M \omega r\right)\left(r \frac{\partial}{\partial r}-M \omega r^{2}\right)+\frac{\partial^{2}}{\partial z^{2}}+\frac{1}{r^{2}} \frac{\partial^{2}}{\partial \phi^{2}}\right] \Psi} \\
& +\frac{\alpha}{4}\left(K_{F}\right)_{\mu \nu \alpha \beta} F^{\mu \nu}(x) F^{\alpha \beta}(x) \Psi=\left(M+S(r)^{2} \Psi .\right.
\end{aligned}
$$

One of the properties of the tensor $\left(K_{F}\right)_{\mu \nu \alpha \beta}$ is that it can be written in terms of $3 \times 3$ matrices that represent the parity-even sector of it : $\left(\kappa_{D E}\right)_{j k}=$ $-2\left(K_{F}\right)_{0 j 0 k}$ and $\left(\kappa_{H B}\right)_{j k}=\frac{1}{2} \epsilon_{j p q} \epsilon_{k l m}\left(K_{F}\right)^{p q l m}$ and the parity-odd sector of this tensor are represented by the $3 \times 3:\left(\kappa_{D B}\right)_{j k}=-\left(\kappa_{H E}\right)_{k j}=\epsilon_{k p q}\left(K_{F}\right)^{0 j p q}$. Note that the matrices $\left(\kappa_{D E}\right)_{j k}$ and $\left(\kappa_{H B}\right)_{j k}$ are symmetric and the matrices $\left(\kappa_{D B}\right)_{j k}$ and $\left(\kappa_{H E}\right)_{k j}$ have no symmetry. In this way, we can rewrite $(2)$ in the form :

$$
\begin{aligned}
& {\left[-\frac{\partial^{2}}{\partial t^{2}}+\frac{\partial^{2}}{\partial r^{2}}+\frac{1}{r} \frac{\partial}{\partial r}-2 M \omega-M^{2} \omega^{2} r^{2}+\frac{1}{r^{2}} \frac{\partial^{2}}{\partial \phi^{2}}+\frac{\partial^{2}}{\partial z^{2}}\right] \Psi} \\
& +\left[-\frac{\alpha}{2}\left(\kappa_{D E}\right)_{i j} E^{i} E^{j}+\frac{\alpha}{2}\left(\kappa_{H B}\right)_{j k} B^{i} B^{j}-\alpha\left(\kappa_{D B}\right)_{j k} E^{i} B^{j}\right] \Psi \\
& =(M+S)^{2} \Psi .
\end{aligned}
$$

Let us consider a possible scenario of the Lorentz symmetry violation determined by $\left(\kappa_{D E}\right)_{11}=$ const, $\left(\kappa_{H B}\right)_{33}=$ const and $\left(\kappa_{D B}\right)_{13}=$ const and the field configuration is given by $[20,21,23,24,25]$ :

$$
\vec{B}=B_{0} \hat{z} \quad, \quad \vec{E}=\frac{\lambda}{2} r \hat{r}
$$

where $B_{0}>0, \hat{z}$ is a unit vector in the $z$-direction, $\lambda$ is a constant associated with a linear distribution of electric charge along the axial direction, and $\hat{r}$ is the unit vectors in the radial direction.

As the metric is independent of time and symmetrical by translations along the $z$-axis, as well by rotations. It is reasonable to write the solution 
to Eq. (6) as

$$
\Psi(t, r, \phi, z)=e^{i(-E t+l \phi+k z)} \psi(r),
$$

where $E$ is the energy of the particle, $l=0, \pm 1, \pm 2, \ldots$ are the eigenvalues of the $z$-component of the angular momentum operator, and $k$ is a constant.

Therefore substituting (3) and (8)-(9) into the Eq. (7), we obtain the following radial wave equation for $\psi(r)$ :

$$
\psi^{\prime \prime}(r)+\frac{1}{r} \psi^{\prime}(r)+\left[\Sigma-\frac{j^{2}}{r^{2}}-\sigma^{2} r^{2}-\frac{\beta}{r}-\delta r\right] \psi(r)=0,
$$

where

$$
\begin{aligned}
\Sigma & =E^{2}-M^{2}-k^{2}-2 \eta_{L} \eta_{c}+\frac{1}{2} \alpha B_{0}^{2}\left(\kappa_{H B}\right)_{33}-2 M \omega, \\
j & =\sqrt{l^{2}+\eta_{c}^{2}}, \\
\sigma & =\sqrt{\frac{1}{8} \alpha\left(\kappa_{D E}\right)_{11} \lambda^{2}+\eta_{L}^{2}+M^{2} \omega^{2}}, \\
\beta & =2 M \eta_{c}, \\
\delta & =2 M \eta_{L}+\frac{1}{2} \alpha \lambda B_{0}\left(\kappa_{D B}\right)_{13} .
\end{aligned}
$$

Now, transforming $\xi=\sqrt{\sigma} r$ into the equation (10), we have

$$
\left[\frac{d^{2}}{d \xi^{2}}+\frac{1}{\xi} \frac{d}{d \xi}+\zeta-\xi^{2}-\frac{j^{2}}{\xi^{2}}-\frac{\eta}{\xi}-\theta \xi\right] \psi(\xi)=0,
$$

where

$$
\zeta=\frac{\Sigma}{\sigma} \quad, \quad \eta=\frac{\beta}{\sqrt{\sigma}} \quad, \quad \theta=\frac{\delta}{\sigma^{\frac{3}{2}}} .
$$

Suppose the possible solution to the Eq. (12) is

$$
\psi(\xi)=\xi^{j} e^{-\frac{1}{2}(\xi+\theta) \xi} H(\xi) .
$$

Substituting the solution (14) into the Eq. (12), we obtain the following equation

$$
H^{\prime \prime}(\xi)+\left[\frac{1+2 j}{\xi}-2 \xi-\theta\right] H^{\prime}(\xi)+\left[-\frac{\Lambda}{\xi}+\Pi\right] H(\xi)=0,
$$


where

$$
\Pi=\zeta+\frac{\theta^{2}}{4}-2(1+j) \quad, \quad \Lambda=\eta+\frac{\theta}{2}(1+2 j) .
$$

Equation (15) is the biconfluent Heun's differential equation [32, 42, 43] with $H(\xi)$ is the Heun polynomials function.

The above equation (15) can be solved by the Frobenius method. Writing the solution as a power series expansion around the origin [44]:

$$
H(\xi)=\sum_{i=0}^{\infty} c_{i} \xi^{i}
$$

Substituting the power series solution into the Eq. (17), we obtain the following recurrence relation

$$
c_{n+2}=\frac{1}{(n+2)(n+2+2 j)}\left[\left\{\eta+\theta\left(n+\frac{3}{2}+j\right)\right\} c_{n+1}-(\Pi-2 n) c_{n}\right] .
$$

With few coefficients are

$$
\begin{aligned}
c_{1} & =\left(\frac{\eta}{1+2 j}+\frac{\theta}{2}\right) d_{0}, \\
c_{2} & \left.=\frac{1}{4(1+j)}\left[\left\{\eta+\theta\left(j+\frac{3}{2}\right)\right)\right\} c_{1}-\Pi c_{0}\right] .
\end{aligned}
$$

The power series expansion $H(\xi)$ becomes a polynomial of degree $n$ by imposing the following two conditions [32]

$$
\Pi=2 n, \quad(n=1,2, \ldots) \quad, \quad c_{n+1}=0 .
$$

By analyzing the first condition, we obtain following equation of the energy eigenvalue $E_{n, l}$ :

$E_{n, l}= \pm \sqrt{M^{2}+k^{2}+2 \eta_{L} \eta_{c}+2 M \omega+\sigma\left\{2\left(n+1+\sqrt{l^{2}+\eta_{c}^{2}}\right)-\frac{\delta^{2}}{4 \sigma^{3}}\right\}-\frac{1}{2} \alpha B_{0}^{2}\left(\kappa_{H B}\right)_{33}}$

Note that Eq. (21) is not the general expression of energy eigenvalues of the relativistic scalar particle. One can obtain the individual energy level and 
wave function one by one by imposing the additional recurrence condition $c_{n+1}=0$ on the eigenvalue problem.

The corresponding radial wave functions are given by

$$
\psi_{n, l}(\xi)=\xi^{\sqrt{l^{2}+\eta_{c}^{2}}} e^{-\frac{1}{2}\left[\xi+\frac{\delta}{\sigma^{\frac{3}{2}}}\right] \xi} H(\xi) .
$$

Now, we evaluate the individual energy level and wave function one by one as in [32]. For example, $n=1$, we have $\Pi=2$ and $c_{2}=0$ which implies

$$
\begin{aligned}
& \Rightarrow \frac{2}{\eta+\theta\left(\frac{3}{2}+j\right)} c_{0}=\left(\frac{\eta}{1+2 j}+\frac{\theta}{2}\right) c_{0} \\
& \Rightarrow \sigma_{1, l}^{3}-\left[\frac{\beta^{2}}{2(1+2 j)}\right] \sigma_{1, l}^{2}-\beta \delta\left(\frac{1+j}{1+2 j}\right) \sigma_{1, l}-(3+2 j) \frac{\delta^{2}}{8}=0
\end{aligned}
$$

a constraint on the parameter $\sigma_{1, l}$, that is, on the oscillator frequency $\omega_{1, l}$. We can see from Eq. (23) that this equation has at least one real root and it is exactly this solution that gives us a first degree polynomial to the Heun function for the radial mode $n=1$. We also see that the allowed values of the oscillator frequency depends on the quantum numbers $\{n, l\}$ of the relativistic system, the Lorentz Symmetry violation parameters, and the potential parameters.

The allowed values of the oscillator frequency for the radial mode $n=1$ is

$$
\omega_{1, l}=\frac{1}{M} \sqrt{\sigma_{1, l}^{2}-\frac{1}{8} \alpha\left(\kappa_{D E}\right)_{11} \lambda^{2}-\eta_{L}^{2}}
$$

The ground state energy level for the radial mode $n=1$ is given by

$E_{1, l}= \pm \sqrt{M^{2}+k^{2}+2 \eta_{L} \eta_{c}+2 M \omega_{1, l}+2 \sigma_{1, l}\left(2+\sqrt{l^{2}+\eta_{c}^{2}}\right)-\frac{1}{2} \alpha B_{0}^{2}\left(\kappa_{H B}\right)_{33}-\left(\frac{\delta}{2 \sigma_{1, l}}\right)^{2}}$

And the ground state wave function is

$$
\psi_{1, l}(\xi)=\xi^{\sqrt{l^{2}+\eta_{c}^{2}}} e^{-\frac{1}{2}\left[\xi+\frac{\delta}{\frac{3}{2}} \sigma_{1, l}^{\frac{3}{2}}\right]}\left(1+d_{1} \xi\right)
$$


where $d_{0}=1$ and

$$
d_{1}=\frac{1}{\sqrt{\sigma_{1, l}}}\left[\frac{M \eta_{c}}{\left(\frac{1}{2}+\sqrt{l^{2}+\eta_{c}^{2}}\right)}+\frac{\delta}{2 \sigma_{1, l}}\right]
$$

Thus by substituting the real solution Eq. (23) into the Eq. (24), we get the lowest energy state eigenvalue Eq. (25) plus the ground state wave function (26)-(27) for the radial mode $n=1$.

\section{Conclusions}

We have investigated the effects of a linear central potential induced by the violation of Lorentz symmetry background on a relativistic quantum oscillator. We have introduced a scalar potential as a modification of the mass term $M \rightarrow M+S(r)$, and then coupled oscillator with scalar particle via the transformation of the momentum operator $\vec{p} \rightarrow(\vec{p}+i M \omega \vec{r})$ in the relativistic wave equation. We have shown that the solution of the bound state to the wave equation can be obtained in a scenario of the Lorentz symmetry violation effects defined by a linear radial electric field, a uniform magnetic field along the $z$-direction, and a tensor background out of the Standard Model Extension possessing the non-null components $\left(\kappa_{D B}\right)_{13}=$ const, $\left(\kappa_{D E}\right)_{11}=$ const and $\left(\kappa_{H B}\right)_{33}=$ const. After solving the oscillator wave equation, the non-compact expression of the energy eigenvalues Eq. (21) and the radial wave function Eq. (22) is obtained. By imposing the condition $c_{n+1}=0$ on the eigenvalue, one can obtain the individual energy level and the wave function, for example, the lowest state energy level Eq. (25) and the corresponding wave function (26)-(27) with the restriction (24) on the angular frequency of the oscillator for the radial mode $n=1$ instead of $n=0$. This effect arises due to the presence of a Cornell-type potential and the Lorentz symmetry violation. We have seen that the angular frequency of the oscillator depends on the Lorentz symmetry breaking parameters and 
the quantum numbers $\{n, l\}$ of the relativistic system. Thus for each energy level, we have a different relation of the angular frequency of the oscillator, so we have labelled $\omega \rightarrow \omega_{n, l}$.

\section{Data Availability}

No data has been used to prepare this manuscript.

\section{Conflict of Interest}

There is no conflict of interest regarding publication this manuscript.

\section{References}

[1] S. Bruce and P. Minning, II Nuovo Cimento A 106, 711 (1993).

[2] V. V. Dvoeglazov, II Nuovo Cimento A 107, 1413 (1994).

[3] M. Moshinsky, J. Phys. A : Math. Gen. 22, L817 (1989).

[4] A. Boumali and N. Messai, Can. J. Phys. 92, 1460 (2014).

[5] J. Carvalho, A. M. M. Carvalho, E. Cavalcante, and C. Furtado, Eur. Phys. J. C 76, 365 (2016).

[6] Z. Wang, Z. Long, C. Long and M. Wu, Eur Phys. J. Plus 130, 36 (2015).

[7] M. Hosseinpour, H. Hassanabadi and Marc de Montigny, Int. J. Geom. Meths Mod. Phys. 15, 1850165 (2018).

[8] F. Ahmed, Int. J. Geom. Meths Mod. Phys. 17, 2050138 (2020).

[9] M. Hosseini, H. Hassanabadi, S. Hassanabadi and P. Sedaghatnia, Int. J. Geom. Meths. Mod. Phys. 16, 1950054 (2019). 
[10] F. Ahmed, Eur. Phys. J. C 78, 598 (2018).

[11] H. Sobhani, H. Hassanabadi and W. S. Chung, Int. J. Geom. Meths Mod. Phys. 15, 1850037 (2018).

[12] F. Ahmed, EPL 130, 40003 (2020).

[13] F. Ahmed, Adv. High Energy Phys. 2020, 5691025 (2020).

[14] V. A. Kostelecky and M. Mewes, Phys. Rev. Lett. 87, 251304 (2001).

[15] V. A. Kostelecky and M. Mewes, Phys. Rev. D 66, 056005 (2002).

[16] H. Belich, F. J. L. Leal, H. L. C. Louzada and M. T. D. Orlando, Phys. Rev. D 86, 125037 (2012).

[17] D. Colladay and V. A. Kostelecky, Phys. Rev. D 55, 6760 (1997).

[18] D. Colladay and V. A. Kostelecky, Phys. Rev. D 58, 116002 (1998).

[19] A. P. Baeta Scarpelli, H. Belich, J. L. Boldo, L. P. Colatto, J. A. HelayelNeto, A. L. M. A. Nogueira, Nucl. Phys. (Proc. Suppl.) 127, 105 (2004).

[20] K. Bakke and H. Belich, Ann. Phys. (N. Y.) 373, 115 (2016).

[21] F. Ahmed, IJMPA (2021), DOI : 10.1142/S0217751X21501281.

[22] K. Bakke and H. Belich, Ann. Phys. (N. Y) 354, 1 (2015).

[23] R. L. L. Vitoria and H. Belich, Eur. Phys. J. C 78, 999 (2018).

[24] F. Ahmed, DOI : 10.20944/preprints202105.0782.v1.

[25] R. L. L. Vitoria, H. Belich and K.Bakke, Adv. High Energy Phys. 2017, 6893084 (2017).

[26] K. Bakke and H. Belich, Ann. Phys. (N. Y.) 360, 596 (2015). 
[27] F. Ahmed, DOI : 10.20944/preprints202105.0755.v1.

[28] R. L. L. Vitoria, H. Belich and K.Bakke, Eur. Phys. J. Plus 132, 27 (2017).

[29] R. L. L. Vitoria, K.Bakke and H. Belich, Ann. Phys. (N. Y.) 399, 117 (2018).

[30] F. Ahmed, DOI : 10.20944/preprints202105.0748.v1.

[31] T. Y. Wu and W. Y. P. Hwang, Relativistic Quantum Mechanics and Quantum Fields, World Scientific Publishing Co. Pvt. Ltd., Singapore (1991).

[32] E. R. F. Medeiros and E. R. Bezerra de Mello, Eur. Phys. J. C 72, 2051 (2012).

[33] M. K. Bahar and F. Yasuk, Adv. High Energy Phys. 2013, 814985 (2013).

[34] M. de Montigny, M. Hosseinpour and H. Hassanabadi, Int. J. Mod. Phys. A 31, 1650191 (2016).

[35] M. S. Cunha, C. R. Muniz, H. R. Christiansen and V. B. Bezerra, Eur. Phys. J. C 76, 512 (2016).

[36] F. Ahmed, Adv. High Energy Phys. 2020, 4832010 (2020).

[37] H. Hassanabadi, E. Maghsoodi and S. Zarrinkamar, Ann. Phys. (Berlin) 525, 944 (2013).

[38] H. Hassanabadi and S. Rahmani, Few-Body Syst. 56, 691 (2015).

[39] H. Hassanabadi, S. Rahmani and S. Zarrinkamar, Phys. Rev. D 90, 074024 (2014). 
[40] C. Alexandrou, P. de Forcrand and O. Jahn, Nuclear Phys. B (Proc. Supp.) 119, 667 (2003).

[41] B. Mirza, M. Mohadesi, Commun. Theor. Phys. 42, 664 (2004).

[42] A. Ronveaux, Heun's Differential Equations, Oxford University Press, Oxford ( 1995).

[43] S. Y. Slavyanov and W. Lay, Special Functions: A Unified Theory Based in Singularities, Oxford University Press, New York (2000).

[44] G. B. Arfken and H. J. Weber, Mathematical Methods For Physicists, Elsevier Academic Press, London (2005). 